\title{
ORIGINAL ARTICLE \\ Outcomes of whole-body computed tomography in spinal cord-injured patients with sepsis
}

\author{
WA Ahmed ${ }^{1}$, LL de Heredia $^{2}$, RJ Hughes ${ }^{2}$, M Belci $^{1}$ and TM Meagher ${ }^{2}$
}

\begin{abstract}
Study design: Retrospective case series.
Objectives: To evaluate the efficacy of body computed tomography (CT) in spinal cord injury (SCI) patients with sepsis.

Setting: Specialist acute care and rehabilitation SCl centre in United Kingdom.

Methods: Patients with SCI and suspected or known sepsis, who had CT of the chest, abdomen and pelvis, over a 4-year period, were identified. Only patients who fulfilled the definition of sepsis or severe sepsis were included. Their medical notes and CT scans were reviewed and clinical outcomes and radiological findings recorded.
\end{abstract}

Results: Twenty-two patients with sepsis were identified including seven categorised as having severe sepsis. A specific radiological diagnosis was found in three patients (14\%) and non-specific findings were found in 15 patients (68\%).

Conclusion: Although in the majority of cases, the findings were non-specific, a small number of patients had significant pathology identified by CT, which influenced their management significantly.

Spinal Cord (2014) 52, 536-540; doi:10.1038/sc.2014.42; published online 15 April 2014

\section{INTRODUCTION}

Patients with spinal cord injury (SCI) have many predisposing factors that make them more susceptible to sepsis than able-bodied individuals. ${ }^{1}$ Indwelling urethral catheters, urinary stasis, renal tract calculi, assisted ventilation and pressure sores are among the risk factors that may lead to significant infections.

Urinary tract infection is the most common cause of sepsis in SCI patients ${ }^{2}$ but making a definite diagnosis of sepsis is more challenging in this population. The presence of urethral catheters and contamination of urinary specimens limits urinary culture and antimicrobial sensitivity in establishing a definite diagnosis of urinary tract infection. ${ }^{2}$ Ventilated patients commonly have areas of atelectasis that may be difficult to differentiate from consolidation on chest X-ray, and limited chest expansion also can limit radiographic evaluation.

The spinal physician must balance history and clinical examination with available laboratory and imaging results to make the best possible diagnosis for the likely cause of infection and begin appropriate treatment. In a small minority of patients, the infection may not respond to treatment and symptoms may progress and deteriorate. In this situation, it may be appropriate to use complex imaging and try to establish a specific diagnosis. ${ }^{2,3}$

Fever in SCI patients may be due to non-infective causes such as thromboembolic disease, drug-induced fever and heterotopic ossification ${ }^{4}$ but the majority $(73-81 \%)$ of all febrile episodes in SCI patients have infectious causes. ${ }^{4,5}$

We examine the use of body computed tomography (CT) to identify the effectiveness in diagnosing the source of sepsis in SCI patients with significant septic episode.

\section{MATERIALS AND METHODS}

This is a retrospective case series study, which includes review of medical records, laboratory results, CT scan reports and reassessment of CT images. Patients having body CT (CT of chest, abdomen and pelvis) with an indication of sepsis were identified from the radiology management system from January 2008 to December 2011.

The CT scans for all the series were performed using the following parameters: (i) CT-chest: $120 \mathrm{kVp}, 100 \mathrm{mAs}, 1.5$-s rotation time, pitch of $1.5,8-\mathrm{mm}$ acquisition, $3 \mathrm{~mm}$ reconstructed slice thickness and $35 \mathrm{~s}$ delay following injection of $75 \mathrm{ml}$ of contrast medium (Xenetix 350, Guerbet Asia Pacific Ltd, Hong Kong, China); and (ii) CT—abdomen/pelvis: $120 \mathrm{kVp}, 160$ $\mathrm{mAs}$, 0.5 -s rotation time, pitch of $1.5,8 \mathrm{~mm}$ acquisitions, 2 -mm reconstructed slice thickness and $60 \mathrm{~s}$ delay after contrast.

Inclusion criteria in this patient cohort were the following: (i) $\mathrm{SCI}$ patients admitted for more than 7 days with unexplained fever $\left(>38.3{ }^{\circ} \mathrm{C}\right)$, (ii) SCI patients who had fever $\left(>38.3^{\circ} \mathrm{C}\right)$ with signs of organ dysfunction or tissue hypoperfusion; and (iii) those who fulfilled the sepsis diagnostic criteria as set by the Trust's Sepsis-Integrated Care Pathway Guideline 59 (Supplementary Data). Patients under active treatment for cancer were excluded from the study.

Medical records reviewed included daily clinical entries, observation charts, blood tests and microbiology reports. The patients' corresponding body CT scans were reviewed in the picture archiving and communication system by two of the study authors (one of them is a radiologist with special interest in spinal injuries)

The CT findings were divided into specific and non-specific radiological findings. The specific findings include those which are regarded as definite causes of sepsis such as pyelonephritis, intra-abdominal collection and bowel perforation. The non-specific findings are outlined in Table 1 and include equivocal radiological abnormalities such as pleural effusion, consolidation, chronic pyelonephritis and splenomegaly.

${ }^{1}$ National Spinal Injuries Centre, Stoke Mandeville Hospital, Aylesbury, UK and ${ }^{2}$ Department of Radiology, Stoke Mandeville Hospital, Aylesbury, UK Correspondence: WA Ahmed, National Spinal Injuries Centre, Stoke Mandeville Hospital, Aylesbury HP21 8AL, UK. E-mail: wailawad2009@gmail.com

Received 6 December 2013; revised 5 March 2014; accepted 16 March 2014; published online 15 April 2014 


\begin{tabular}{|c|c|}
\hline \multicolumn{2}{|l|}{ Nonspecific CT findings } \\
\hline Site & Finding \\
\hline \multirow[t]{4}{*}{ Chest } & Consolidation \\
\hline & Ground glass appearance \\
\hline & Bilateral small pleural effusions \\
\hline & Atelectasis \\
\hline \multirow[t]{3}{*}{ Urinary tract } & Chronic pyelonephritis \\
\hline & Enlarged kidney \\
\hline & Ureteric stones \\
\hline \multirow[t]{2}{*}{ Hip joint } & Effusion \\
\hline & Heterotopic ossification \\
\hline \multirow[t]{2}{*}{ Lympho-reticular system } & Axillary and inguinal lymphadenopathy \\
\hline & Splenomegaly \\
\hline Miscellaneous & Ovarian dermoid cyst \\
\hline
\end{tabular}

A full color version of this Table is available at the Spinal Cord journal online.

\section{RESULTS}

One of 23 patients reviewed was excluded because of active nonHodgkin's lymphoma leaving 22 patients included in the study. Fifteen patients had sepsis and seven patients were classified as having severe sepsis. The mean age was 40 years (range: 14-76 years) and male to female ratio was 10:1. Twelve patients were tetraplegics and 10 paraplegics. Seventeen had complete SCI (American Spinal Injury Association Impairment Scale; (AIS) A), and five patients had incomplete SCI (AIS B, C or D). Before body CT, all patients had a recorded temperature of greater than or equal to $38.3^{\circ} \mathrm{C}$, apart from one patient who was hypothermic with a temperature of $<36.0^{\circ} \mathrm{C}$ (Table 2).

The final clinical diagnosis was pneumonia in 10 cases, empyema, intraperitoneal abscess, septicaemia secondary to urinary tract infection, ureteritis with ureterocoele, urinary tract infection and heterotopic ossification in single individual cases. In six patients, no clinical diagnosis was established.

A specific radiological cause was identified in three cases (14\%; Figures 1-3) and non-specific imaging findings were noted in 15 cases. The following were the three cases with specific radiological findings:

(1) Patient 1: intraperitoneal abscess where the CT scan demonstrated intraperitoneal free gas and a dislodged intraperitoneal gastrostomy tube (Figures 1a and b). The patient's condition settled following a laparotomy operation with re-fashioning of the gastrostomy.

(2) Patient 2: ureteritis where the CT scan demonstrated an enhancing ureter (Figure 2a). Following treatment with antibiotics, the patient's condition did not improve and subsequent ultrasound and CT urogram showed evidence of ureterocoele as an underlying cause (Figures $2 \mathrm{~b}$ and c). The patient's condition settled after cystoscopic drainage and insertion of J-J stent.

(3) Patient 3: pneumonia with the CT showing typical lower lobe peribronchovascular air space shadowing typical of active infection (Figure 3).

There were no radiological abnormalities in four cases (18\%). Correlation between the clinical diagnosis and radiological findings (including specific, non-specific and no abnormalities) was found in 12 patients (55\%).

Most of the patients' episodes of sepsis settled receiving medical treatment and few settled following surgical interventions apart from one patient who died in the intensive therapy unit following acute respiratory distress syndrome.

\section{DISCUSSION}

Sepsis is defined as infection plus systemic manifestations and severe sepsis is defined as sepsis plus sepsis-induced organ dysfunction or tissue hypoperfusion. ${ }^{6}$ The initial diagnostic pathway following clinical suspicion of sepsis includes microbiology samples (for blood, urine, sputum, wound swab cultures), inflammatory markers, differential blood count and imaging studies including chest X-ray and abdominal ultrasound.

Imaging of suspected occult sepsis is a common diagnostic situation in hospital patients. Ultrasound is frequently performed initially to exclude hydronephrosis, obvious intra-abdominal collection and pleural effusion (which might not be visible on chest X-ray) but published guidance ${ }^{6,7}$ indicates that CT remains the best overall imaging test in this situation and is the current standard. 
Table 2 The sepsis diagnostic criteria of all 22 patients

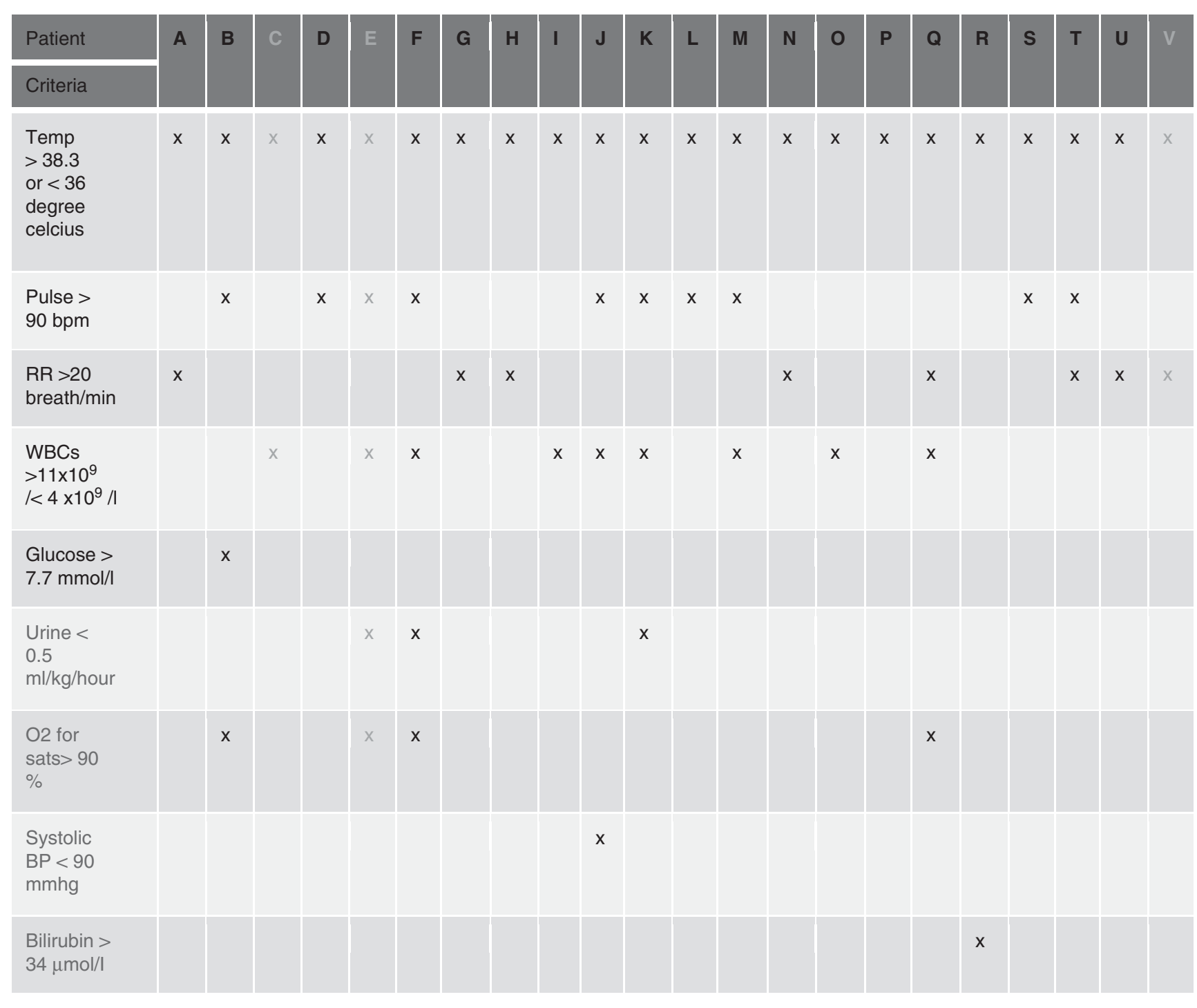

Abbreviations: $\mathrm{BP}$, blood pressure; bpm, beats per minute; RR, respiratory rate

Patients with specific findings are patients $C, E$ and V. Criteria of severe sepsis are the last four.

A full color version of this Table is available at the Spinal Cord journal online.

In a patient with persisting sepsis, ultrasound would generally be first line to identify obvious pathology including intra-abdominal collection, lung pathology or bowel inflammatory conditions. Magnetic resonance imaging is less appropriate in the clinical situation where the site of possible infection is not clinically evident. Imaging the chest, abdomen and pelvis with the most current magnetic resonance imaging systems is a lengthy examination and is outside the current clinical guidelines. ${ }^{6,7}$

CT scan is a more comprehensive examination to identify the likely source of sepsis. It is also widely used in sepsis cases with suspected post-operative intraperitoneal collections. Furthermore, in severely septic patients, therapeutic percutaneous drainage (using CT scan) of deeply seated infections is preferred to surgical drainage as it is a less invasive approach. ${ }^{8}$

The clinical problem of sepsis is common in admitted patients with SCI, whether during initial hospitalisation following cord injury or subsequent re-admission. The most common causes of infection are related to the urinary tract and in patients with assisted ventilation lower respiratory tract infection is also prevalent.

Owing to various pathophysiological changes that occur as a result of SCI, tetraplegic and paraplegic patients constitute a different group of patients to non-SCI patients. For instance, in cases of acute abdomen, sensory loss is a primary reason for delay in diagnosis as tenderness and rebound tenderness might not be elicited. An important sign of acute abdomen like abdominal musculature guarding might be masked by spasticity. Abdominal distension is common in SCI patients because of constipation, faecal impaction and intestinal fermentation. Unless it happens suddenly and progresses, it might be unremarkable. Furthermore, the degree of SCI (complete or incomplete) and the different levels do not give a typical picture to spot the diagnosis. In addition to that, autonomic 

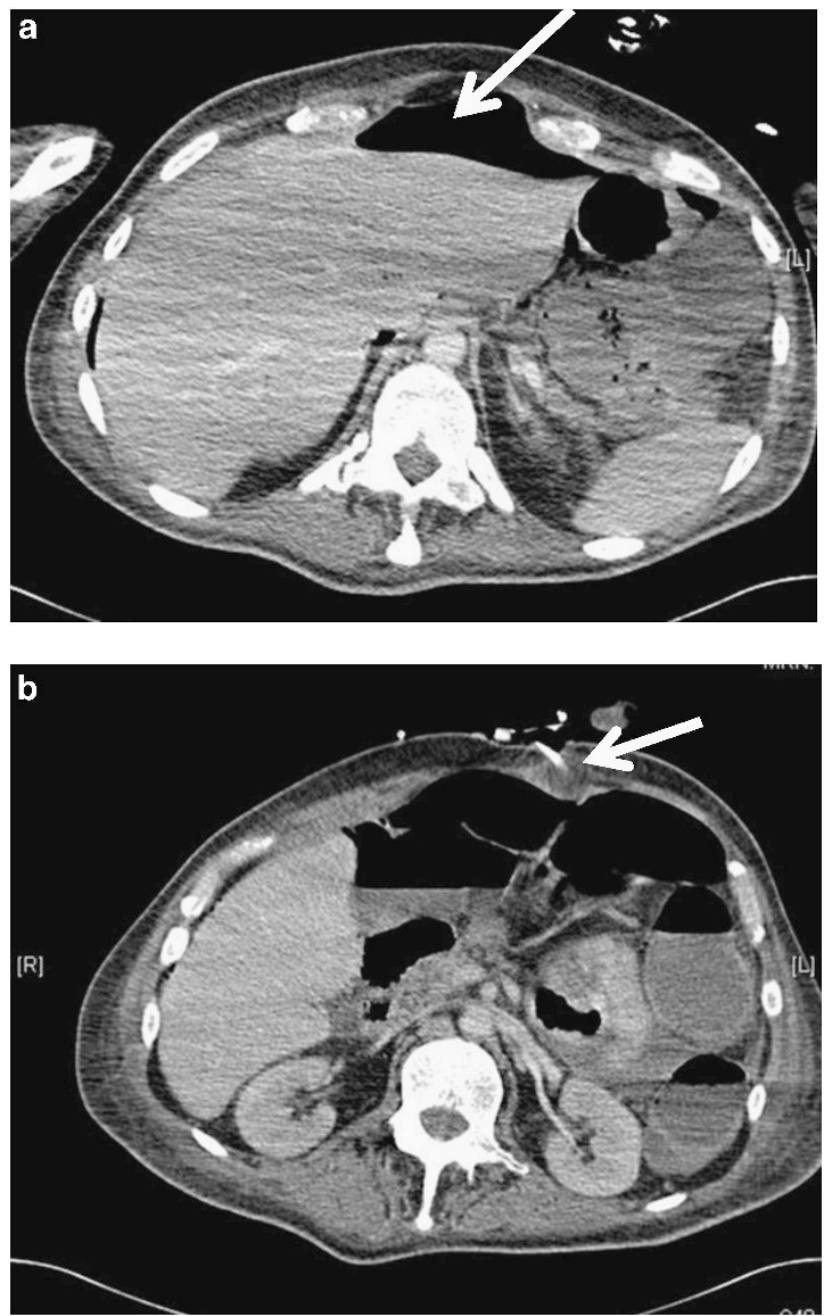

Figure 1 (a) Showing intraperitoneal free gas (white arrow). (b) Showing a balloon of a dislodged gastrostomy tube (white arrow). dysreflexia, in patients paralysed at T6 and above, might complicate the picture.

It may be difficult to prove conclusively with laboratory testing as to the precise cause of infection. Most frequently, symptoms and signs of infection, in SCI patients, resolve following clinical diagnosis and commencement of appropriate treatment. In a small number, symptoms will persist and even deteriorate with some patients developing signs of severe sepsis requiring circulatory support.

In this clinical situation when clinical examination, routine laboratory investigations and basic imaging (usually chest X-ray and abdominal ultrasound) are unhelpful in defining the cause of infection, it is reasonable to proceed to body CT.

CT is widely available and provides evaluation of common causes of sources of infection including the renal tract, respiratory system while also defining collections and complications related to spinal fixation. Radiation doses are significant (compared with normal background annual radiation of $2 \mathrm{mSv})^{10}$ in this relatively young population but in this scenario the benefits outweigh the risks. As technology progresses, radiation dose with CT is falling dramatically

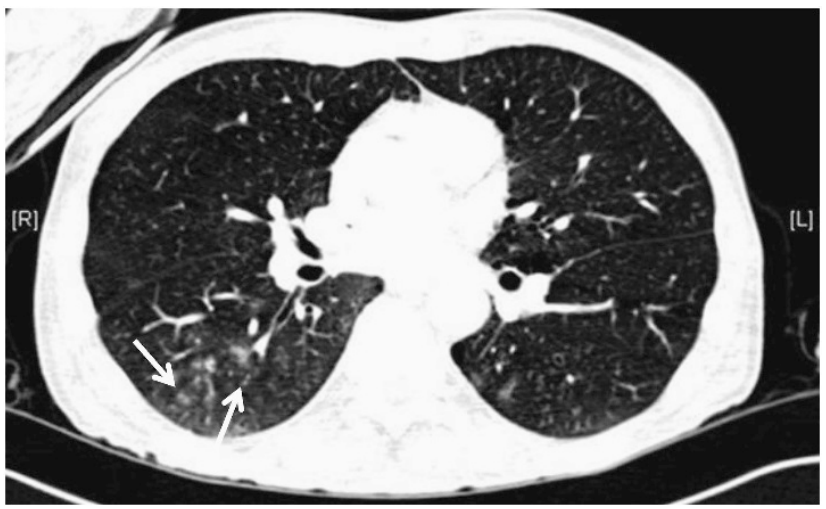

Figure 3 Showing peribronchovascular nodules in the apical segment of right lower lobe in keeping with infection (white arrows).
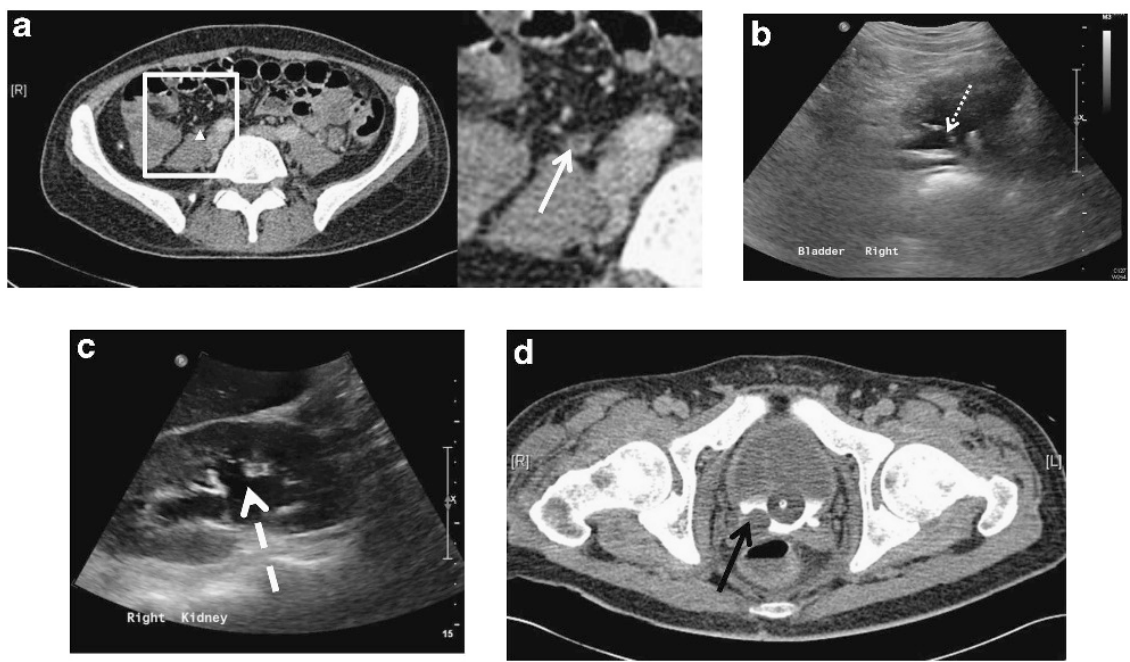

Figure 2 (a) CT scan showing enhanced right ureter suggestive of ureteritis (white arrow head and white arrow in magnified image). (b) Ultrasound scan (USS) showing right ureterocoele (dotted white arrow). (c) USS showing right hydronephrosis (dashed white arrow). (d) CT urogram showing ureterocoele (black arrow). 
and this factor may become of less importance, particularly for unwell patients.

The exam will require intravenous iodinated contrast and it will be necessary to check renal function to avoid contrast-related nephropathy.

The small number of cases in this series indicates that severe sepsis in SCI patients in a tertiary institution (typically 150 new cases of SCI per annum and 800 re-admissions) is not common. Although a specific diagnostic yield of $14 \%$ is not high, the elucidation of a specific cause that requires intervention is important and may prevent worsening morbidity.

Non-specific findings such as consolidation, atelectasis and pleural effusion are common on CT, particularly in ill patients. It is important that there is close dialogue between the clinical team and radiologists to ensure a good understanding of the importance of clinical signs outlined on CT.

In summary, whole-body CT is an appropriate investigation for patients with severe sepsis when initial laboratory and imaging are unhelpful. Close liaison between clinicians and radiologists is necessary to ensure accurate interpretation of positive findings on CT.

\section{DATA ARCHIVING}

There were no data to deposit.

\section{CONFLICT OF INTEREST}

The authors declare no conflict of interest.

1 Darouiche R. Infection and Spinal Cord Injury. In: Vernon W (ed.). Spinal Cord Medicine: Principles and Practice, 2nd edn. Demos Medical: NY, USA, 2010, pp 263-269.

2 de Kleijn EM, Vandenbroucke JP, van der Meer JW. Fever of unknown origin (FUO) I A. prospective multicenter study of 167 patients with FUO, using fixed epidemiologic entry criteria. Medicine (Baltimore) 1997; 76: 392-400.

3 Varghese GM, Trowbridge P, Doherty T. Investigating and managing pyrexia of unknown origin in adults. BMJ 2010; 341: C5470.

4 Beraldo P, Neves E, Alves C, Khan P, Cirilo A, Alencar M. Pyrexia in hospitalised spinal cord injury patients. Paraplegia 1993; 31: 186-191.

5 Sugarman B, Brown D, Musher D. Fever and infection in spinal cord injury patients. JAMA 1982; 248: 66-70.

6 National Guideline C. ACR Appropriateness Criteria\&reg; Acute (nonlocalized) Abdominal Pain and Fever or Suspected Abdominal Abscess. Agency for Healthcare Research and Quality (AHRQ): Rockville, MD, USA, 2014. Available from: http:// www.guideline.gov/content. aspx?id=37926.

7 Radiologists RCo. IRefer: Making the Best Use of Clinical Radiology. The Royal College of Radiologists: London, UK, 2012.

8 Dellinger RP, Levy MM, Rhodes A, Annane D, Gerlach H, Opal SM. et al. Surviving sepsis campaign: international guidelines for management of severe sepsis and septic shock: 2012. Crit Care Med 2013; 41: 580-637.

9 Eltorai I, Jakowatz J, Juler G. The acute abdomen. In: Eltorai I (ed.). Emergencies in Chronic Spinal Cord Injury Patients, 3rd edn. Eastern Paralyzed Veterans Association: NY, USA, 2001, pp 67-79.

10 Brenner DJ, Elliston CD. Estimated radiation risks potentially associated with full-body CT screening. Radiology 2004; 232: 735-738.

Supplementary Information accompanies this paper on the Spinal Cord website (http://www.nature.com/sc) 